\title{
LA MÚLTIPLE DIMENSIÓN DE LA PRECARIEDAD LABORAL: EL CASO DE LA ADMINISTRACIÓN PÚBLICA EN ARGENTINA ${ }^{1}$
}

\author{
THE MULTIPLE DIMENSION OF LABOR PRECARIZATION: \\ THE CASE OF PUBLIC ADMINISTRATION IN ARGENTINA
}

\author{
Nicolás Diana Menéndez*
}

RESUMEN

En este trabajo se problematiza la definición comúnmente utilizada de precariedad laboral y se propone un abordaje multidimensional, a partir del análisis del proceso de fragmentación y precarización laboral que se produjo en la Administración Pública en Argentina, producto de las reformas del Estado durante los años noventa. La estrategia metodológica seguida utiliza elementos de orden cualitativo y cuantitativo. En las conclusiones se sostiene la necesidad del abordaje multidimensional de la precariedad para poder dar cuenta de la complejidad y extensión del proceso de vulnerabilidad del mundo del trabajo.

PALABRAS CLAVE: ARGENTINA * EMPLEO * SECTOR PÚBLICO * PRECARIEDAD LABORAL * ADMINISTRACIÓN PÚBLICA * ESTADO * SINDICATOS

\section{ABSTRACT}

This article problematizes the usually definition of labor precarization and it proposes a multidimetional approach, by analyzing the precarization of labor process that occurred in the public administration in Argentina underwent since the Nineties. The methodology utilized was qualitative and quantitative. In the conclusions, we proposed the need to tackle the precarization as multidimensional to understand the complexity and extension of vulnerability in world's work.

KEY WORDS: ARGENTINA * EMPLOYMENT $*$ PUBLIC SECTOR $*$ LABOR PRECARIZATION * PUBLIC ADMINISTRATION $*$ STATE $*$ TRADE UNIONS

El presente trabajo forma parte de una investigación mayor que se desarrolla desde hace cinco años y que se plasmó en la tesis de doctorado "Condiciones de trabajo y precarización laboral en el Estado".
Investigador del Centro de Estudios e Investigaciones Laborales/Programa de Investigaciones Económicas sobre Tecnología, Trabajo y Empleo (CEIL-PIETTE/Conicet, Argentina). ndiana74@yahoo.com.ar 


\section{INTRODUCCIÓN}

Si bien existe una vasta literatura que se ocupa de la problemática de la precariedad laboral, en general se le asume como un compartimiento estanco, como poseyendo un carácter fijo, es decir, considerando que un trabajo es o no precario.

La intención en este artículo es problematizar esa definición de trabajo precario explorando las distintas dimensiones de vulnerabilidad laboral identificadas durante la investigación de campo, dando lugar a una complejización de las situaciones de precariedad. Particularmente se propone examinar las dimensiones objetivas de la precariedad laboral, analizándola como se presenta en este singular campo de estudio, el Estado. Para ello, será necesario remontarse al proceso que comenzó en la década del noventa con las reformas del Estado, momento del congelamiento de la planta permanente $y$ de la implementación de una diversidad de contratos precarizados en la Administración Pública Nacional en Argentina (en adelante: APN).

El universo al que se circunscribe la presente investigación es el de los organismos centralizados del Ministerio de Trabajo, Empleo y Seguridad Social de la Nación Argentina (en adelante MT). En este estudio se utilizan herramientas metodológicas cualitativas y cuantitativas, a fin de triangular los datos. Se revisaron $y$ analizaron estadísticas $y$ documentos oficiales $y$ de otros organismos, $y$ se realizaron más de 40 entrevistas en profundidad entre el 2005 y el 2008 , tanto a trabajadores pertenecientes a las diferentes modalidades de contratación que se desempeñan en el Ministerio de Trabajo, también se realizaron entrevistas de control en el Ministerio de Educación, como a dirigentes sindicales y funcionarios encargados de la gestión de los recursos humanos de la $\mathrm{APN}^{2}$.

2 Se realizaron entrevistas a funcionarios de Recursos Humanos de los ministerios y a funcionarios de la Subsecretaría de la Gestión Pública.

\section{EL CONCEPTO DE PRECARIEDAD LABORAL}

Los análisis en torno a la precariedad laboral están estrechamente vinculados a los de la flexibilidad laboral. Tanto es así, que de algún modo, representan dos caras de la misma realidad, y están atravesadas por cosmovisiones ideológicas fuertes. Originariamente el concepto de flexibilidad se presentaba en el marco de las teorías posfordista (neoshumpeteriana, regulacionista y especialización flexible) como una nueva modalidad de movilización de la fuerza de trabajo vinculada a las innovaciones toyotistas de la organización del trabajo, que aparecían como el nuevo paradigma que reemplazaría al modelo fordista. Ideas como polivalencia, trabajo en equipo, autonomía, movilidad interna, cliente interno, calidad, constituía parte del vocabulario novedoso que se imponía (Coriat, 1992; Novick, 2000; Battistini, 2001). La noción de flexibilidad asociada al toyotismo, implicaba así una flexibilidad negociada al interior del proceso productivo.

Ahora bien como señala De la Garza (2007), en Latinoamérica la "flexibilidad amable" (negociada y enriquecedora del trabajo) que supuestamente venía asociada a los cambios en el régimen de acumulación, se desvaneció frente a la realidad de un claro predominio de flexibilizaciones no pactadas sino impuestas a sindicatos $y$ trabajadores, al cambiarles sus contratos colectivos en detrimento de estos últimos, dando lugar a una "flexibilización en general con unilateralidad gerencial". Así la implementación de las políticas flexibilizadoras, en los hechos, operó produciendo condiciones precarias a los trabajadores en todas las latitudes en que fueron aplicadas y particularmente en Argentina.

El origen del concepto de precariedad laboral data de hace tres décadas como significado de determinadas situaciones "atípicas" de empleo. Su antecedente inmediato está vinculado a los debates en torno a la marginalidad que se desarrollaron durante la década del 70 , de gran gravitación en las realidades latinoamericanas. Estrechamente asociado a las conclusiones de este debate, surgió la caracterización de una parte marginal del aparato productivo muy 
particular, el sector informal. Ante la insuficiencia explicativa de las categorías de la informalidad para dar cuenta de los procesos de privaciones por parte de trabajadores vinculados a lo que es considerado sector formal, se instaló la conceptualización de la precariedad laboral (Pok, 1992; De la Garza, 2007).

En los años ochenta, la OIT delimitó el concepto de empleo precario a partir de la carencia de ciertas dimensiones pertenecientes a las denominadas Condiciones y Medio Ambiente de Trabajo (CYMAT ${ }^{3}$ ), a partir de la ausencia de "salario indirecto" y "estabilidad en el empleo". En su libro de 1983, El trabajo en el Mundo, aparecen algunas definiciones referidas al trabajo no protegido $y$ al trabajo precario o clandestino (Orsatti, 1989). Por otra parte la OIT también ha asociado la categoría al empleo ilegal, diferenciando empleo precario legal e ilegal (vg. trabajo sexual, recicladores, etc.).

El autor pionero de la conceptualización del empleo precario, Guy Caire (1982: 137-140), sostuvo que el empleo precario se caracteriza por una doble afectación de la naturaleza del contrato y de la identificación del empleador. Para este mismo autor, las formas en que puede presentarse el empleo precario son: los contratos de duración determinada, el trabajo en negro, algunas formas de trabajo a domicilio, trabajos eventuales, los mediados por agencias de trabajo temporarios, la subcontratación, los part time, aprendices, etc.

Otros trabajos publicados en la misma década siguen las propuestas de la OIT como los de Córdova (1985) y Galin y Feldman (1990). Córdova habla del trabajo atípico, e identifica como formas principales: "los temporarios, otras formas de subcontratación, préstamos de trabajadores entre empresas, trabajo a tiempo parcial, trabajo estacional, sucesión ininterrumpida de relaciones de trabajo a domicilio

3 Los estudios de las CYMAT refieren a las condiciones generales en que se realiza el trabajo, incluyendo tanto la organización, la calidad e intensidad del mismo, el tipo de actividad, etc. Rodolfo Capón Filas (1994:23) la define como "todos los elementos reales que inciden directa o indirectamente en la salud de los trabajadores; constituyen un conjunto que obra en la realidad concreta de la situación laboral". $y$ trabajo clandestino" 4 . Por su parte Galin $y$ Feldman (1990: 9-10) sostienen que en la mayor parte de la bibliografía la conceptualización se define por defecto, es decir, el empleo precario se define por su oposición a las formas típicas. Se considera típico al trabajo de tiempo completo, que sea ejercido en un lugar único donde el asalariado está ligado también a un empleador único y protegido por la legislación o la negociación colectiva. El universo del empleo precario sería entonces el del trabajo clandestino, la subcontratación, los contratos a plazo, las prácticas de agencias de trabajo temporal, el empleo clandestino, el empleo asalariado fraudulento, etc.

Rodgers (1992), otro autor pionero de la definición de la noción de precariedad, señala que se tiende a considerar precarias las formas de trabajo cuando se desvían de la norma del trabajo regular estable. El autor distingue cuatro dimensiones de precariedad:

$\diamond \quad$ En primer lugar, el grado de certidumbre respecto a la continuidad del trabajo, asî serían precarios los trabajos de horizonte a corto plazo, y los de riesgo de pérdida elevados;

$\diamond \quad$ en segundo lugar, una dimensión referente a los aspectos del control sobre el trabajo, de este modo, cuanto menos control por parte del trabajador más inseguro es el trabajo;

$\diamond \quad$ en tercer término, la protección legal individual y colectiva del trabajador;

$\diamond \quad y$ finalmente, la dimensión referida a la insuficiencia de los ingresos. Así para este autor, la condición de precariedad implica la conjunción de estas cuatro dimensiones anteriores, de manera que para Rodgers "un trabajo inestable no necesariamente tiene que ser precario"5 (1992: 19).

$4 \quad$ Citado por Orsatti, 1989.

5 Si bien en esa afirmación, el autor señala que para encontrarse frente a una situación de precariedad deben darse concurrentemente estas cuatro dimensiones, en el mismo texto, poco más adelante, relativiza dicha afirmación al sostener — coincidentemente con el abordaje que se realiza aquí"esto sugiere que un modelo dual, que distinga entre trabajadores seguros y precarios, puede, en 
Por su parte, Cingolani (2005) argumenta que existen tres sentidos de la precariedad: el trabajo/empleo precario, el salario precario $y$ la precariedad de la vida asociada a la pobreza; Paugam (2000) destaca las precariedades del trabajo y la del empleo, señalando que pueden superponerse. En el 2007, Bouffartigue tomó a los dos autores mencionados y estableció tres formas de precariedad que pueden presentarse juntas o no. En primer lugar, señala la precariedad en el empleo vinculada al contrato de trabajo - precariedad de derecho-y es un componente de la inestabilidad en el empleo - precariedad de hecho-. En segundo lugar, consigna la precariedad del trabajo, asociada más directamente a las condiciones generales y cotidianas del trabajo, como el reconocimiento práctico y simbólico. En tercer y último término, enuncia las "faltas" referidas a las relaciones laborales, que tiene que ver con la ineficacia o la débil aplicación del derecho del trabajo y del derecho sindical.

Como se observa, la noción de precariedad o precarización posee una diversidad de definiciones que se caracterizan por la negativa. Estas definiciones son variadas, más amplias o más estrechas, más jurídicas (Córdova, 1980; Meik y Zas, 1999) o más sociológicas (Feldman y Galin, 1990; Marshall, 1990; Lindemboin, 2001; Bonofiglio y Fernández, 2004; Salvia, 2003 ; etc.) pero todas conservan una línea común que las atraviesa y las hacen parte de un conjunto: el carácter de negatividad que inmediatamente connota este concepto, y que evidentemente no es inocente sino que, por el contrario, está asociado a concepciones ideológicas fuertes que acuerdan en colocar en la centralidad de las problemáticas al trabajo $y$ fundamentalmente a quienes lo llevan adelante, los trabajadores.

En términos generales la precariedad puede nombrarse como un incremento de la vulnerabilidad de los trabajadores tanto en la

determinadas situaciones, ser menos útil que una preocupación más general por los grados de precariedad $y$ de vulnerabilidad que puedan producirse entre grupos de trabajadores o bien afecten a toda la fuerza de trabajo en conjunto" (Rodgers, 1992: $20)$. estabilidad como en la calidad de las condiciones de trabajo. Esta vulnerabilidad merma la capacidad y autonomía de proyección y planificación de su propia vida por parte de los trabajadores, comportando a su vez, una mayor asimetría de las relaciones de poder entre capital y trabajo (Cano, 1998). La perspectiva aquí adoptada — como se verá- no asumirá la diferenciación estricta entre trabajadores precarios y no precarios. Resulta más exacto hablar de dimensiones de precariedad que se encuentran presentes en diversos grados $y$ modalidades en gran parte de las formas de empleo.

\section{SOBRE EL EMPLEO EN LA ADMINISTRACIÓN PÚBLICA}

En este apartado se realizará una breve aproximación al marco histórico y jurídico vigente para el empleo en la Administración Pública, antes de continuar con el análisis. En el marco de las políticas neoliberales que se implementaron en Argentina durante la década del 90 en el gobierno presidido por Carlos Menem, se llevó a cabo una transformación intensa del Estado ${ }^{6}$. Entre las medidas que impactaron en el empleo público se cuenta las privatizaciones de empresas públicas, medidas de reducción de las plantillas como los retiros voluntarios, firma de dos convenios colectivos de trabajo ${ }^{7}$, un nuevo

$6 \quad$ Respecto de las reformas implementadas durante la presidencia de Carlos Menem (1989-1999) pueden diferenciarse dos etapas tanto en términos temporales como por los objetivos que se propusieron. Las primeras reformas provocaron un cambio ostensible del rol del Estado, reduciendo significativamente su injerencia en la esfera económica y social, vía privatización de funciones estatales clave, transferencia de los monopolios públicos a manos privadas, desregulación y descentralización que modificaron sustancialmente las relaciones entre la esfera pública y la privada. Las reformas denominadas de segunda generación tuvieron por objeto la "refuncionalización" del Estado, buscando modificar su fisonomía y comportamiento interno.

$7 \quad$ En el año 1999 durante la presidencia de Menem y con la sola signatura del sindicato UPCN se firmó el primer convenio colectivo de la Administración Pública Nacional y en 2005, ya con Néstor 
escalafón para la función pública $\left(\right.$ SINAPA $\left.^{8}\right)$ y el congelamiento de las vacantes en la planta estable del sector público con el consecuente ingreso de empleados públicos con contratos temporarios y precarizados.

Dicho congelamiento de vacantes producido en 1994 impuso inexorablemente la necesidad de incorporar personal a través de contratos. Con ese fin se promulgó el decreto 92/95 que reglamentaba la incorporación transitoria de los profesionales y técnicos, mediante contratos anuales, asimilándolos de este modo a la figura de trabajador autónomo profesional de la actividad privada. Las contrataciones que se realizaban bajo este régimen tendrían por objeto la prestación de servicios técnicos o profesionales especializados y/o la ejecución de obras, sean ellos de carácter individual o comprendidos en programas de trabajo, tanto de fortalecimiento institucional como de proyectos especiales de estudios e investigación, cuya realización hubiera sido evaluada y aprobada por la máxima autoridad de la Jurisdicción (Orlansky, 1997).

También se habilitaban por entonces, las horas cátedra y las pasantías universitarias, que representan otras modalidades de incorporar personal de modo precario. Existen asimismo los contratos a través de fondos especiales, que se implementan en algunos organismos de la APN (ej.: entes cooperadores del Ministerio de Justicia, responsables de recaudación a través de los sistemas registrales), que poseen recaudación propia y han generado mecanismos particulares de contratación. Muchos de sus empleados trabajan en una sede administrativa del Estado, pero cobran un salario distinto al del resto de los trabajadores estatales y su relación contractual está regida por la Ley de Contrato de Trabajo (CENDA, 2007).

Kirchner en el poder, se rubricó el segundo convenio, esta vez refrendado por las dos organizaciones sindicales. Durante 2008 se firmó un tercer convenio colectivo, bajo la presidencia de Cristina Fernández de Kirchner.

8 Sistema Nacional de Profesión Administrativa. A fines de 2008 este sistema fue reemplazado por otro denominado Sistema Nacional de Empleo Público (SINEP).
Finalmente, se instrumentan los convenios de costos compartidos con organismos internacionales, de este modo el Estado empleador contrata a través de los programas cuando es el propio Estado el que realiza los pagos salariales, conformándose una tercerización de hecho.

Unos años después se firmó el primer convenio colectivo (con uno solo de los dos sindicatos que poseen injerencia a nivel nacional), y se promulgó el decreto $1184 / 2001^{9}$ que reemplazó al 92/95. Como señalan Orlansky y otros (2007:10), dicho decreto incluye a aquellos contratos cuyo objeto fuera la prestación de servicios especializados técnicos o servicios profesionales a título personal, y no la realización de actividades administrativas. Así, contempla las figuras de "coordinador general", "consultor" y "asistente técnico" cuyas tareas estuvieran más relacionadas con el objetivo de un resultado esperado en el marco de proyectos de complejidad variable que con el cumplimiento de una tarea predeterminada. A su vez, según dispone el decreto mencionado, "la actividad a realizar por el contratado podrá sufrir modificaciones para ser adecuada a las variaciones que pueda experimentar el desarrollo de los objetivos para los que fue contratado y el mejor logro de estos", lo cual supone la flexibilidad del contratado.

Actualmente, el sistema se rige por la ley marco del empleo público nacional $(25$ 164) reglamentada por el decreto $1421 / 2002$. En estas dos normas están contemplados los trabajadores de la planta permanente ${ }^{10}$ (agentes públicos cabalmente), y los contratados en virtud del decreto 1421/02 quienes constituyen una planta de trabajadores con características "híbridas", dado que poseen casi todas las mismas prerrogativas que la planta permanente, con excepción de la estabilidad, ya que son

$9 \quad$ Este decreto fue reemplazado en enero de 2009 por el decreto 2345/08 donde se establecen nuevos perfiles para los profesionales y un nuevo régimen retributivo.

10 El ingreso a la planta permanente de la APN fue congelado en 1994, y recién en 2008 comenzaron a abrirse concursos en número significativo, pero que aún son incipientes. 
contratos con plazo de vencimiento máximo de un año.

La realidad es que desde el congelamiento de la planta permanente en 1994, los trabajadores que ingresaron a la Administración Pública debieron hacerlo bajo alguno de los regímenes contractuales mencionados, pasando muchos de estos trabajadores de uno a otro tipo de contrato a lo largo de los años. Es necesario destacar que el régimen contemplado en el decreto 1421/02 comenzó a hacerse efectivo de modo significativo entre 2005 y 2006; y que la amplia mayoría de los trabajadores que hoy se rigen por él vienen de los otros regímenes de contratación. Por tanto y más allá de las falencias que comporta, este régimen constituye un proceso creciente de reconocimiento de vínculos de dependencia laboral.

Para realizar una evaluación cronológica es pertinente observar los números publicados por la Secretaría de Hacienda del Ministerio de Economía, para todo el empleo del poder ejecutivo (no solo las áreas centralizadas). Allí puede verse que para diciembre del año 2001 el poder ejecutivo contaba con 16816 trabajadores con- tratados bajo las modalidades 1184/01, mientras que casi cinco años después, para el segundo trimestre de 2006 (último período relevado), la cifra asciende a 23676 personas contratadas (incluyendo las modalidades 1184/01 y 1421/02). Este aumento considerable se explica no solo por el ingreso de nuevos trabajadores a las plantillas del Estado, sino fundamentalmente por el proceso de relativo "blanqueo" (o más estrictamente: reconocimiento de la relación laboral) que se llevó a cabo en la Administración Pública a lo largo de los años 2005 y $2006^{11}$. En efecto, mediante el traspaso a la modalidad del decreto 1421/02 se van incorporando a una modalidad que reconoce el vinculo laboral con el Estado, trabajadores que previamente se vinculaban con él vía contratos que desconocían esa relación como el 1184 y otros trabajadores no reconocidos como los ya mencionados contratos por PNUD y otros terciarizados.

$11 \quad$ Sobre los años 2007 y 2008 no se encuentran datos del MECON, aún así el proceso de pases a esa modalidad continúa.

CUADRO 1

DATOS SOBRE EL PODER EJECUTIVO NACIONAL SOBRE EL TOTAL DEL PERSONAL PERMANENTE/TRANSITORIO Y CONTRATADO, EN TÉRMINOS TOTALES Y PORCENTUALES ENTRE DICIEMBRE DE 2001 Y JULIO DE 2006

\begin{tabular}{llll}
\hline \multirow{2}{*}{ TRIMESTRE } & $\begin{array}{l}\text { PERSONAL PERMANENTE } \\
\text { Y TRANSITORIO TOTAL Y } \\
\text { PORCENTUAL }\end{array}$ & $\begin{array}{l}\text { PERSONAL CONTRATADO } \\
\text { TOTAL Y PORCENTUAL }\end{array}$ & TOTAL \\
\hline Diciembre 2001 & $226361(93 \%)$ & $16816(7 \%)$ & $243177(100 \%)$ \\
Diciembre 2002 & $227111(93,4 \%)$ & $16601(6,6 \%)$ & $243172(100 \%)$ \\
Diciembre 2003 & $227160(92,7 \%)$ & $17859(7,8 \%)$ & $245019(100 \%)$ \\
Diciembre 2004 & $229443(92,2 \%)$ & $19276(7,8 \%)$ & $248719(100 \%)$ \\
$4^{\text {a }}$ cuatrimestre de 2005 & $238200(91,5 \%)$ & $22082(8,5 \%)$ & $260282(100 \%)$ \\
$2^{\text {a }}$ cuatrimestre de 2006 & $238652(91 \%)$ & $23676(9 \%)$ & $262328(100 \%)$ \\
\hline
\end{tabular}

Fuente: Ministerio de Economía. En:<http://www2.mecon.gov.ar/hacienda/ocupacion/principal.htm> 
Si bien no se cuenta con información previa al año 2001, es altamente presumible que los contratos han cumplido, desde su implementación (masiva) en los albores de la década del noventa, un rol sustancial, en la medida en que se constituyeron en la modalidad prácticamente exclusiva de ingreso al sector público. Es elocuente en ese sentido la relación que, por ejemplo, en el Poder Ejecutivo hay entre el total de trabajadores y el total de contratados considerados según los datos oficiales. Un valor cercano al $10 \%$ de la totalidad de los trabajadores del Poder Ejecutivo es muy relevante, máxime si se toma en cuenta que no están contempladas todas las modalidades de contratación.

Las estimaciones presentadas en el cuadro siguiente muestran la distribución de trabajadores bajo los distintos regímenes en el organismo específicamente analizado.

CUADRO 2

MINISTERIO DE TRABAJO, ÁREAS CENTRALIZADAS DE TODO EL PAÍS

\begin{tabular}{cc}
\hline Planta permanente (1) & 968 \\
Contratado decreto 1421 (1) & 1868 \\
Régimen de pasantías educativas (2) & 18 \\
Contratados decreto 1184 (1) & 93 \\
Contratado financiamiento externo (PNUD y otros) (2) & 455 \\
Contrato de Asistencia Técnica (2) & 70 \\
\hline Total & 3472 \\
\hline
\end{tabular}

Fuentes: (1) Subsecretaría de la gestión pública y (2) estimaciones en base a datos propios. Salvo los datos de la planta permanente que corresponden a 2006, el resto corresponden todos a 2007.

Las fuentes son variadas en virtud de la escasez de datos oficiales sobre tipos de vinculación, por lo que hubo que complementarlas con datos propios recabados en esta investigación a partir del cruce de datos brindados por distintos actores. Aquí se puede observar que sumando el resto de las categorías de contratos no contempladas en el cuadro anterior, la diferencia entre trabajadores contratados y estables es muy significativa conformando una relación de 3 a 1.

\section{DIMENSIONES DE LA PRECARIEDAD}

En las aproximaciones a los procesos de precarización del trabajo suelen encontrarse análisis que tratan esta problemática desde un punto de vista netamente objetivo, que suele asumir la precariedad como un estado dado o no, como una condición fija. Como se mencionó, la precariedad que en términos generales puede nombrarse como un incremento en la vulnerabilidad de los trabajadores (ya material, ya simbólica); posee por eso mismo una serie de dimensiones que pueden analizarse $y$ detectarse en los distintos segmentos del "mercado de trabajo" interno del Estado. De este modo, es más certero hablar de dimensiones de precariedad laboral que están asociadas al deterioro general de las condiciones de trabajo que afectan, por una u otra vía, al conjunto de los trabajadores, incluso en el Estado; dado el marco de las relaciones de fuerza entre el capital $y$ el trabajo en que se inscribe $y$ que allí se expresa (Poulantzas, 1980).

Analizando y desmenuzando los procesos y realidades laborales puede distinguirse ocho dimensiones de precariedad que afectan la realidad de los trabajadores contratados de la Administración Pública Argentina, lo que permite observar esa condición en distintos grados según los casos: 
1. la falta de estabilidad en el empleo

2. el deterioro de las condiciones de trabajo que implican una mayor degradación $y$ vulnerabilidad

3. la incertidumbre e insuficiencia del ingreso

4. la reducción de la protección y seguridad social (Cano, 1994, 1996)

5. el deterioro del reconocimiento práctico y simbólico de la realidad del trabajo (Boufartigue, 2007)

6. la inestabilidad política del vínculo laboral, dimensión que atañe particularmente al universo aquí analizado

7. la vinculada al incremento de la heterogeneización laboral y en consecuencia la mayor fragmentación y el subsiguiente deterioro de los lazos y vínculos al interior del colectivo de trabajo (y el proceso de individualización)

8. $y$ finalmente, la falta de representación $y$ cobertura sindical.

\section{LA PRECARIEDAD ENTRE LOS TRABAJADORES CONTRATADOS}

Como es sabido, el Estado no es un organismo unánime y unívoco, que responde acabadamente a las directivas de un poder omnímodo y coherente. Por el contrario, cada una de sus dependencias cristaliza en su interior una relación de fuerzas sociales (Poulanztas, 1980). En el Estado, si bien guarda una coherencia general, en el sentido de que pese a las resistencias que se le oponen, los grandes lineamientos de los proyectos políticos suelen ponerse en marcha, coexisten diversas lógicas que alteran sustancialmente las planificaciones, por más exigentes y pormenorizadas que sean. De este modo, se produjo respecto de las contrataciones de personal a lo largo de la Administración, la coexistencia de una lógica general y de múltiples lógicas particulares en cada uno de los organismos e incluso en cada oficina.

El universo de los trabajadores contratados no es uniforme. A partir de su diversidad se conforma una heterogeneización compleja de sensaciones, identidades, relaciones, perspectivas, lógicas de acción, etc. Se puede comenzar a desenredar esta madeja realizando una clasificación de tres categorías de trabajadores contratados originarios ${ }^{12}$.

En primer término, los funcionarios contratados con alta relación con el poder político de turno, que suelen ocupar funciones directivas y acceder a sueldos elevados (dentro de lo que es el promedio del sector público). Estos funcionarios, ingresaron masivamente al MT durante los primeros años noventa, a partir de la "colonización" de sectores de este ministerio por parte del Ministro de Economía de entonces Domingo Cavallo. De este modo desembarcaron muchos profesionales formados $y$ en muchos casos con altos niveles de consenso respecto de las políticas de reforma que estaba llevando adelante el gobierno. La implementación del contrato, permitía de este modo, incorporar discrecionalmente personal con los perfiles requeridos, con altos ingresos, sin necesidad de alterar toda la política salarial de la Administración. También a través de los contratos se pagaban favores políticos y personales.

Un segundo grupo se incorporó al MT y al Estado en general, en una suerte de cuadros medios. En términos generales, estos trabajadores eran profesionales, o estudiantes universitarios de carreras relacionadas con las Ciencias Sociales y la Economía, y que cobraban buenos salarios, que superaban en mucho los salarios percibidos por los trabajadores estables. También ellos cumplían con los requisitos de profesionalización de la gestión, aunque en este caso, no necesariamente formaban parte de la "tropa" política, sino que fue la única forma de ingreso posible a estas labores. No obstante, estos trabajadores también se representaban como una nueva burocracia, que pretendía distinguirse de la existente. Más que como trabajadores, al menos en un principio, la imagen del consultor se encarnaba mejor en su imaginario que la de empleado público, con toda la carga

12 Se impone señalar que estas distinciones que señalamos comenzaron a achicarse en la medida en que se comenzó a hacer efectivo el pase al régimen del decreto 1421/02, y que un conjunto mayor de trabajadores fueron incorporándose. No obstante al día de hoy existen significativas diferencias entre los diversos grupos. 
estigmatizada que representa. En el siguiente relato de un delegado sindical se da cuenta de esa imagen.

Los mismos contratados, durante un tiempo bastante largo, particularmente en la época de oro de la convertibilidad, venían con una concepción mental muy del orden neoclásico, mejor dicho, neoliberal y se consideraban ser algo diferente que empleados públicos. O sea, que si bien por un lado, desde la perspectiva de los empleados de planta los discriminábamos como contratados, ellos también como contratados, creían ser algo diferente... mejor y de más calidad que el personal de planta del ministerio (delegado de ATE del MT, Planta permanente).

Finalmente, entre los contratados, debe hacerse mención a los que accedieron al trabajo del modo más precario. Estos trabajadores, si bien pueden estar más o menos formados, en general eran estudiantes universitarios, que ingresaron al MT a realizar tareas de carácter menos técnicas, más cercanas a lo administrativo. En estos casos, muchos eran pasantes que realizan tareas menos calificadas, tales como cadetería, recepción, call center, etc. (lejos de las condiciones de formación que la norma de pasantías exige). Estos trabajadores, encarnaban las condiciones de mayor precariedad, puesto que ni siquiera contaban con el beneficio de un salario promedio.

Una cuestión esencial a destacar, luego de hecha esta clasificación de trabajadores, es la siguiente: tanto la figura del consultor como la del pasante, constituyen y consagran una negación de la relación laboral. Esto conlleva varias cuestiones significativas. En primer lugar, un problema de orden legal o jurídico, se constituye un fraude ${ }^{13}$, en la medida en que se

13 Una salvedad necesaria es que, en principio, las pasantías no constituyen un fraude puesto que existen como modalidad de incorporación, sin embargo gran parte de las condiciones que deben cumplir para ser consideradas tales, son omitidas con lo cual también se encubre una relación laboral de dependencia. Entre ellas puede mencionarse los plazos vencidos, el tipo de tareas que nada niega una relación de dependencia que de hecho existe. En segundo término, desde el punto de vista sociológico, el desconocimiento del vínculo contiene muchas consecuencias. Una de ellas es la diferenciación que se impone producto de la distinción entre trabajadores por una lado (los de planta) $y$ "consultores" $y$ pasantes por el otro.

La noción de consultor merece un tratamiento específico, ya que contiene una carga distintiva de gran magnitud, es un concepto fuerte que puede ser muy atractivo para ciertos sectores sociales, en el marco de la sociedad "globalizada". La primera ruptura con el pasado, con lo que se quiere dejar atrás, es la ruptura conceptual y discursiva, la forma de nombrar y de nombrarse. La idea de consultor refiere mucho más a un profesional, autónomo, moderno, flexible, en definitiva, remite inmediatamente al "encumbrado" profesional $y / 0$ a las grandes y exitosas consultoras económicas $y$ de otras disciplinas que han proliferado en los últimos años, a la vez que se distancia de la idea de empleado público. Asimismo, se asocia más férreamente a una idea de vinculación individual, rompiendo con la lógica de referencia colectiva que está históricamente asociada a la noción de trabajador. Considerando además que buena parte de estos "consultores" son jóvenes profesionales o estudiantes universitarios, en muchos casos sin experiencia laboral anterior, el ropaje de consultor es "un sayo que les cabe".

\section{FALTA DE ESTABILIDAD LABORAL}

Evidentemente el punto de partida tiene que ver con la cuestión que los define como contratados: su vínculo laboral con el Estado, es decir, su inestabilidad. Como se mencionó, existen una serie de modalidades contractuales mediante las cuales ejercer laboralmente en la

\footnotetext{
tienen que ver con la carrera universitaria, etc. Por otra parte, existen fallos judiciales que obligan a reconocer la relación laboral, y por tanto al pago de indemnizaciones por despido. Ver por ejemplo: Sentencia Causa nro. 37.217/02 sala VI-Cámara Nacional de Apelaciones del Trabajo. Juzgado nro. 7. Autores: Rodríguez Rodríguez, Juan Carlos c/.Ministerio de Economía y Obras y Servicios Públicos y otro, s/despido.
} 
Administración Pública: pasantías, contratos de asistencia técnica, contratos vía PNUD y otros organismos, contratos del decreto 1421/02, contratos del decreto 1184/01, etc. A la fecha, $y$ según los últimos datos disponibles, en el MT alrededor de un $60 \%$ de los trabajadores se encuentran enmarcados dentro de lo dispuesto en el decreto 1421/02. Este último, si bien adopta muchas de las condiciones correspondientes al trabajo registrado, adolece de una falta sumamente significativa como es la estabilidad. Y este es justamente el hecho que hoy iguala a todos los trabajadores contratados, su inestabilidad. Luego, los grados de vulnerabilidad de unos y otros varían ostensiblemente. Como se sostuvo en el anterior apartado, una primera distinción sustancial es que algunas de las modalidades reconocen un vínculo laboral y la condición de trabajo en relación de dependencia, y otras establecen una relación de carácter "comercial" entre trabajador y empleador, violentando el vínculo.

Así, por una parte, se establecen vínculos laborales y comerciales con los trabajadores, quedando estos últimos desamparados del derecho laboral. Y por otra parte, los trabajadores contratados en su conjunto pueden poseer distintos empleadores, para unos es el Estado Nacional; para otros, algún otro organismo o fundación y para unos terceros, ningún empleador.

\section{DETERIORO DE LAS CONDICIONES DE TRABAJO}

Si se remite a las condiciones generales de trabajo, en términos de su degradación y vulnerabilidad, también puede encontrarse un proceso de individualización de las condiciones, en donde las inasistencias, las vacaciones, las licencias por enfermedad, los horarios, etc., son variables dependientes de la coordinación o dirección de cada oficina o de cada organismo. Si bien se han extendido criterios generales respecto de todas estas cuestiones que establecen "usos comunes", no obsta que muchas condiciones queden sujetas a la arbitrariedad (más positiva o más negativa) del "uso" de cada oficina. En efecto, el proceso de individualización de las condiciones en que se realizan las tareas, somete a cada trabajador a definir los modos de acceder (o no) a determinados derechos laborales no contemplados explícitamente en sus contratos, en función de las vinculaciones individuales (mejores o peores) que pueda $y$ sepa establecer con los superiores que le tocaran en suerte. Del mismo modo, los propios jefes de las oficinas se encuentran resolviendo situaciones de carácter laboral que no le competerían (al menos con tanta injerencia) en una situación de empleo formalizada. Un trabajador, en condición de pasante, relataba lo siguiente:

—Digamos, hoy en día, el hecho de irme de vacaciones depende más del que... no sé... aparentemente tendría 14 días por el tema de la pasantía, días por estudios que los puedo utilizar para las vacaciones; ahora cuando yo voy $y$ hablo nadie sabe si realmente es así...(...) El hecho de estar en planta te da otra estabilidad; yo qué sé, yo estoy acá adentro y cada tanto hay una bajada de línea: si no te gusta la situación acá te podés ir... vos sabés que acá nadie te ata, el vínculo no está estable... De hecho me lo han dicho varias ya... (trabajador contratado del MT, 28 años).

Muchas de estas falencias han ido salvándose con la implementación de la modalidad del artículo 9 de la ley marco, que reconoce buena parte de estos derechos ausentes en el resto de las modalidades. Respecto de las 'cymat' (condiciones y medioambiente de trabajo) debe decirse que los trabajadores contratados están sometidos a las mismas condiciones que los permanentes, en donde, en términos generales, cunden las falencias. Sin embargo, cabe mencionar que entre organismos (entre Ministerios por ejemplo), e incluso al interior de cada uno (entre secretarías por ejemplo), pueden encontrarse diferencias ostensibles vinculadas al peso relativo que se ostente en cada período histórico.

\section{INCERTIDUMBRE E INSUFICIENCIA DE LOS INGRESOS}

En tercer término, respecto de la dimensión vinculada a la incertidumbre e insuficiencia 
de ingresos, se observa que estos trabajadores, fundamentalmente quienes vienen laborando en el MT desde el gobierno de Carlos Menem han visto modificarse muchas de sus condiciones de trabajo; entre las que prima la sustancial mengua de ingresos que sufrieron con las bajas en los montos de los contratos y la reducción del 12\% que afectó a todo el personal. En términos generales estas personas vieron reducidos sus haberes en un 50\%. El testimonio de un trabajador contratado que lleva varios años en esa condición es elocuente al respecto:

-Yo me acuerdo, a mí me bajaron primero... cuánto fue, el $12 \%$... cuando llegaron los de la Alianza, a los que no rajaron, nos bajaron el sueldo. Después del $12 \%$ a todo el mundo y después me bajaron el 30\%, así que imagínate.... yo me acuerdo... yo pasé a ganar... cuando llegaron ellos ganaba 1500 mangos... y pasé a ganar $800 . .$. (...) Lo más gracioso de todo que cuando hacen la devolución del 12\% a los contratados no se los hacen... (trabajador contratado del MT, 32 años).

Del mismo modo que a la baja, para poder percibir aumentos salariales no existe para los contratados reglas claras, con cada renovación de contrato existe la posibilidad, pero depende casi exclusivamente de la construcción individual de cada uno, la relación con su jefe, y la voluntad de este. En este sentido hay que mencionar que en la práctica, pese a esta falta de reglas, los contratados tuvieron mayores posibilidades reales de acceder a una suba salarial anual que los trabajadores de la planta que están sujetos a los (escasísimos) concursos. Actualmente, los aumentos negociados por convenio otorgan cierta movilidad salarial aunque no de escalafón.

Como se observa, la situación que se presenta es que la incertidumbre y la falta de reglas para los aumentos de los contratados los deja sujetos a las vinculaciones personales y a la voluntad política de conseguir recursos de cada oficina. Situación que puede operar, según el caso, más favorable o desfavorablemente.
4. REDUCCIÓN (O INEXISTENCIA) DE LA PROTECCIÓN Y SEGURIDAD SOCIAL

Otra dimensión de la precariedad es la señalada como reducción (o inexistencia) de la protección social, seguros, asignaciones familiares, jubilación, obra social, etc., cabe mencionar que los trabajadores contratados carecen de estos beneficios (salvo quienes pasaron al decreto 1421/02, dado que este régimen contempla muchos de estos beneficios, como los aportes jubilatorios y la obra social). En ese sentido, estos últimos se encuentran en mejor situación que los primeros.

-Y aparte que a nosotros supuestamente te obligan a pagar el monotributo ${ }^{14}$ y si no lo pagás no tenés obra social. Yo tengo obra social porque la pago aparte, pero no pago el monotributo hace como un año por ejemplo. Y a alguien que no se puede pagar la obra social si le pasa algo en el Ministerio, no tiene obra social, es un problema. Ya pasó que quisieron exigir que se presente el comprobante de monotributo y tampoco pudieron... (trabajador contratado del MT, 25 años).

Aparece aquí nuevamente la cuestión del monotributo que constituye todo un problema para quienes deben afrontarlo y no cuentan con buenos salarios, puesto que pagarlo implica una reducción del sueldo de bolsillo y constituye la única forma de acceder a algún tipo de obra social $y$ de aporte jubilatorio. Por otra parte, al incumplir el pago se va generando una deuda personal con el fisco, situación que sufre buena parte de los trabajadores monotribustistas. Extremando el argumento, podría hallarse la situación de que a un trabajador monotributista se le rescinda el contrato, sin "corresponderle" ninguna indemnización y hallarse como deudor del fisco, es decir, del propio Estado empleador

14 El "monotributo" es una figura tributaria unificada y económica, orientada a trabajadores por cuenta propia que poseen bajos montos de facturación. En la realidad del mercado laboral argentino, esta figura es muy utilizada para invisibilizar vínculos de dependencia laboral. 
que le negó el reconocimiento del vínculo laboral $y$ en consecuencia el pago de los aportes a la seguridad social y de la indemnización.

\section{DETERIORO DEL RECONOCIMIENTO PRÁCTICO Y SIMBÓLICO}

En relación con la dimensión del reconocimiento, dimensión estrechamente ceñida a los aspectos subjetivos y experienciales de los actores, puede señalarse como uno de los elementos característicos de la vida laboral en el seno de la Administración Pública, la falta de reconocimientos en términos generales. En efecto, los mecanismos a los que suelen estar asociados tanto los premios como los castigos, que tienen que ver con la movilidad en el escalafón, y los aumentos de erogaciones, poseen una existencia virtual. La falta de concursos reales obtura cualquier desarrollo de carrera, $y$ el reconocimiento material vía erogaciones salariales está sujeto a presupuestos y limitaciones férreas y múltiples.

Por otra parte, la condición de contratado negó (y lo continúa haciendo en muchos casos) el estatuto de trabajador a quienes se vinculaban de ese modo y en consecuencia negó tal reconocimiento. En la imagen señalada del consultor, del gerente, etc., se desvanecen a la vez, el vínculo real legal que establecen empleador y empleado; $y$ la condición de trabajador, con toda la carga social, histórica y política que este estatuto conlleva, entre ellas, como veremos inmediatamente, la referencia al colectivo.

-... lo que estábamos reclamando era más que nada el reconocimiento de nuestra situación como trabajadores de este ministerio, independientemente que no todos sabían por ahí que era prestar servicios en relación de dependencia, porque esto por ahí es una cuestión más técnica, o más vinculada por ahí con aquellos sectores que manejan esta normativa y de más, o la normativa laboral si se quiere. Pero era mejorar nuestras condiciones en general: los ingresos, la cobertura social, empezar a tener apor- tes, uno pensaba desde ya en nuestra futura jubilación (trabajador contratado del MT, 36 años).

Este trabajador pone de manifiesto en su relato, a partir de una experiencia de conflicto en su lugar de trabajo, la falta del reconocimiento al estatuto de trabajador por un lado, pero también señala la falta de reconocimiento ligada a las omisiones de los derechos laborales.

\section{INESTABILIDAD POLÍTICA DEL VÍNCULO LABORAL}

Con la identificación de esta dimensión de la precariedad laboral se pretende dar cuenta de un aspecto que atañe específicamente al sector público, a saber: nos referimos a la inestabilidad política y su relación con la incertidumbre laboral en general. La afectación de una en otra se presenta al menos de dos formas identificables. En primera instancia, como se mencionó ya, la expresada en la memoria de los trabajadores contratados que ingresaron antes del arribo al gobierno de la Alianza, cuando acusados de menemistas fueron echados de sus puestos muchos trabajadores contratados que no poseía más vinculación con el gobierno saliente que el hecho de haber ingresado al empleo durante el periodo de gobierno. Si algo se les ha cristalizado como reflejo es el temor a la pérdida del trabajo reactualizado en cada cambio de gobierno. Esto constituye una cuestión indefectible entre aquellos funcionarios que asumen cargos políticos, ya que por definición, saben que su vida laboral está sujeta a la suerte de la gestión (al igual que los cargos electivos). El problema deviene cuando con el mismo criterio se rescinden los contratos de los trabajadores en funciones "no políticas". Esta problemática se actualizó poco tiempo atrás en el gobierno de la ciudad con la asunción de Mauricio Macri en dicho cargo ${ }^{15}$.

15 En el año 2008, la nueva gestión del gobierno de la ciudad de Buenos Aires, rescindió abruptamente el contrato a 2000 trabajadores contratados con el pretexto de que eran funcionarios "acomodados" que no trabajaban. Luego de varias intervenciones sindicales y judiciales, el gobierno debió revisar sus posiciones. 
En segunda instancia, la incertidumbre laboral ligada al recambio político, se manifiesta también en las condiciones generales de trabajo. Cuando un nuevo gobierno asume, suele plantearse modificaciones en la burocracia a su cargo, tanto en formas como en contenidos, y la experiencia neoliberal de las últimas décadas quedó sellada en la memoria de los trabajadores. Achicar el gasto, reducir plantillas, redireccionar partidas, redefinir mecanismos $y$ rutinas, crear y modificar organismos, etc., todos ítems comunes en las flamantes agendas gubernamentales.

Resta aún analizar dos de las dimensiones señaladas, que serán especialmente consideradas aquí, en virtud de su centralidad, y que están atravesadas por procesos históricos que las han vuelto sumamente dinámicas y significativas. La primera de ellas es la referida al proceso de heterogeneización de los colectivos de trabajo y la consecuente individualización laboral, y lo que en términos históricos constituyó una reversión (relativa) de tal proceso. La segunda dimensión, es la vinculada a la tutela sindical, y que en este caso asume características empíricas e históricas muy particulares, partiendo de la base de que en el ámbito de la APN se encuentran dos sindicatos cohabitando y disputando la representación de los trabajadores del sector.

7. EL PROCESO DE HETEROGENEIZACIÓN E INDIVIDUALIZACIÓN DE LOS COLECTIVOS DEL TRABAJO

La propensión a lo individual, que la precarización y la fragmentación impone posee varias aristas. En primer lugar, se establecen dos grandes grupos (en este caso contratados $y$ planta permanente), dos identidades que se encuentran en conflicto, lo nuevo contra lo viejo, lo eficiente contra lo ineficiente, lo gestionario contra lo punteril, etc.

En segundo término, se impone -apuntalando lo antedicho- una lógica propia del contrato que tiene sus efectos sobre las personas con ese tipo de vinculación. Es la lógica potenciada de la estrategia individual ${ }^{16}$. Los contratos que se firman son individuales, no existen normas en función de antigüedad, desempeño, capacitación, incremento salarial, etc. Todas ellas dependen de la relación que cada trabajador establezca con su superior y de la buena o mala voluntad de este. Como herramienta disciplinaria el contrato es insuperable, la continuidad laboral se asienta en la renovación periódica, en la buena conducta. Un trabajador comenta su visión a partir de su experiencia:

-Y al principio, sí... al principio era siempre esta cosa que te renovaran el contrato, digamos, a ver... está muy supeditado la relación que podés establecer con tus superiores inmediatos $y$ a que la tarea que desarrollás, sea de tu agrado, digamos. Porque en la medida que sos apto para lo que hacés, $y$ te gusta, $y$ tenés buena relaciones con los jefes, podés tener un colchoncito, que sabés que... de alguna manera, las renovaciones siempre van a estar (trabajador contratado del MT, 41 años).

La heterogeneidad contractual, impone una diferenciación entre trabajadores que atraviesa gran parte de los aspectos de su vida laboral. Como recién mencionamos, las trayectorias laborales internas de cada uno están supeditadas a las relaciones que sepa $y / 0$ puedan establecer individualmente, sin ningún criterio de carrera, de este modo se individualizan al extremo estas trayectorias y las reglas de juego son tan volátiles como puedan serlo las voluntades, oportunidades o condiciones de los funcionarios responsables del área. La propia permanencia en un puesto de trabajo, está sujeta a las habilidades relacionales y laborales del trabajador para "reubicarse" cuando un programa financiado externamente se termina. Las

16 Si bien en este trabajo se está lejos de sostener que esto produzca inhibición a la acción colectiva o a la lógica colectiva, (una amplia bibliografía y nuestros propios datos lo niegan rotundamente) si se considera que hay lógicas que dificultan la referencia colectiva. 
acciones que esta lógica fragmentaria impone son la búsqueda de la supervivencia individual, en el marco de un mundo cada vez más individualizado.

Los salarios, de la misma manera, son una función de la relación de la oficina o área en que se encuentre con el poder de turno; $y$ del tipo de relación que establezca con sus superiores (también es esperable que jueguen criterios de eficiencia y esfuerzo). Cada renovación contractual puede ser una suba o una baja de haberes percibidos. En definitiva, esta fragmentación contractual, reproduce el hecho fragmentario en todos los órdenes de la vida laboral (trayectorias, tareas, ingresos, etc.), reduce las relaciones a su existencia individual, lo que en suma implica obturar lo colectivo. Y la destrucción de lo colectivo

... se trata no sólo de una desmotivación, sino también de una real fractura operada en un tejido social en el trabajo, donde se juega en reciprocidad la cuestión de la utilidad de todos, en las particularidades de cada uno. Ahora bien, este tejido social restringido es un terreno irremplazable para el aprendizaje o perfeccionamiento del saber vivir en el tejido social común, fuera del trabajo (Dessors, 1998:82).

Ahora bien, uno de los fenómenos ocurridos cuando se produjo el ingreso post congelamiento de la planta, fue la igualación (en términos de percepción homogénea) de todos los contrata$\mathrm{dos}^{17}$, sin discriminar las diferencias — que se han marcado más arriba - al interior de este "grupo". Es decir, por parte del resto de los involucrados en estas particulares relaciones laborales (incluyendo los trabajadores estables, los funcionarios de diferentes gestiones $y$ los sindicatos), se veía a todos los contratados como privilegiados, haciendo caso omiso de sus diferencias en cuanto a condiciones laborales y generales.

17 Lo mismo puede decirse de parte de los contratados hacia los trabajadores de planta. Estos últimos tampoco conforman un grupo homogéneo, es evidente. Pero en términos de condiciones laborales, están todos sujetos a las mismas normas.
La realidad, es que el universo de quienes estaban contratados era sumamente heterogéneo y no estuvo exento de conflictos a su interior. Las diferencias podían llegar a ser notorias, desde todos los puntos de vista. Pese a estas diferencias entre los trabajadores contratados, para el resto del personal constituían un todo inescindible. Se produjo entonces, un proceso profundo, complejo y duradero de fragmentación de los trabajadores del Estado. La tendencia dominante contemplaba $-y$ aún contempla en alguna medida - la constitución de un nosotros y un ellos en la relación entre trabajadores contratados $y$ de planta permanente. Condiciones de carácter objetivas y otras más subjetivas contribuyeron a facilitar este fenómeno, que trajo aparejada la fragmentación de los intereses de los trabajadores del Estado, dificultando pensarse como conjunto. Dos testimonios brindan una muestra de las visiones cruzadas y aún vigentes entre estos grupos:

—No, no, ahí hay bronca y se nota. Más, digo, en términos de bronca, más de los de planta a los contratados; los contratados a los de planta yo diría más que bronca, desprecio... (trabajador contratado del MT, 35 años).

- O sea, lamentablemente se dio eso pero bueno, eso fue producto de la época, del encantamiento - mal entendido no- porque yo en ningún momento reniego de que vos tenés que estar formado y tenés que estar preparado y tenés que estar reconocido y demás, pero resulta que terminaron reconociéndolos mucho más y en muchos casos obteniendo mejoras económicas superiores a las nuestras, que acá se puso de manifiesto cuando aparece el artículo noveno (trabajador de Planta del MT, 62 años).

Las tensiones y conflictos (a veces explícitos, a veces solapados) atravesaron los espacios de trabajo de modo significativo, nadie quedó exento de las percepciones y prejuicios cruzados que se desplegaron entre los trabajadores de la planta permanente y los contratados. El "otro" 
era en parte culpable, ya de la propia situación (los contratados para los permanentes), ya de la situación del Estado (los de planta para los contratados).

Ahora bien, es evidente que la profunda huella que marcaron estas tensiones y conflictos, continúa presente en la cotidianeidad laboral, pese a que se han ido limando muchas asperezas. A este último hecho concurrieron, la paulatina pérdida de ventajas relativas por parte de los trabajadores contratados (pérdidas dinerarias, despidos, riesgo evidente a perder el puesto), así como la implementación de la llamada resolución 48 de la Subsecretaría de la Gestión Pública (decreto 1421/02), que asimiló a muchos contratados al escalafón de la planta permanente.

Otro factor, no menos importante, que interviene en la dilución paulatina del conflicto entre trabajadores, es el cambio de sensaciones $y$ percepciones entre unos y otros. A lo largo de tantos años de convivencia, es inevitable un proceso de homogeneización de percepciones recíprocas. La cotidianidad, el conocimiento, el lazo generado entre trabajadores en la tarea diaria erosiona la potencialidad de la distinción. Muchos de los prejuicios previos respecto al "otro", se desploman en función de esa convivencia cotidiana.

El hecho de la heterogeneización de las relaciones laborales constituye, desde la perspectiva aquí asumida, una nueva "ausencia", una nueva vulnerabilidad y en consecuencia, una nueva dimensión de la precariedad, al menos por dos motivos. En primero lugar, por la corrosión del vínculo de esa pequeña comunidad cotidiana que es el colectivo de trabajo, como lugar fundamental de sociabilidad $y$ conformación de identidades. Y en segundo término, porque por ese mismo motivo, se convierte en un obstáculo para la acción colectiva (sindical o no) para la definición y defensa de sus intereses.

Esta dimensión de la precariedad laboral afecta a todo el conjunto de los trabajadores, en la medida en que esa "pequeña comunidad" que se ve perjudicada está compuesta por trabajadores contratados y de la planta. Del mismo modo, la corrosión de esa comunidad dificulta la identificación de intereses comunes entre estos dos grupos de trabajadores.

\section{FALTA DE REPRESENTACIÓN Y COBERTURA SINDICAL}

Sobre dos aspectos se profundizará en este apartado, uno de carácter conceptual y otro de carácter histórico. Para comenzar se señalará la ausencia de representación sindical como una dimensión central de la precariedad laboral. Si se considera que, al menos en las sociedades en que la relación capital-trabajo es fundante y estructurante, la forma sindical es una instancia esencial de definición y defensa de los intereses de los trabajadores (Offe y Weissenthal, 1985), la falta de acceso a esta modalidad conforma un elemento significativo de precariedad en sí mismo. Si a ellos se suma que la representación sindical constituye la forma más eficaz y reconocida (legalmente) de acceder a mejores condiciones de trabajo, es decir, que de algún modo es una condición sustancial para conquistar mejoras en el resto de las dimensiones definidas, puede otorgarse a esta última un carácter central (sin por ello quitarle relevancia al resto de las dimensiones identificadas). En consecuencia, es dable pensar que la presencia de esta dimensión eleva en intensidad potencial —aún más- la incidencia de las demás.

En el ámbito de la Administración Pública Nacional, los trabajadores contratados no tuvieron cobertura sindical formal hasta la aparición de la resolución 48 que habilitó el ingreso de algunos trabajadores a la modalidad del artículo 9 de la ley Marco del empleo público. Recién a partir de la vigencia de esa normativa pudieron acceder, aquellos que pasaron a ese tipo de contrato, a la tutela sindical legalmente reconocida.

En este ámbito, dos sindicatos poseen alcance nacional, disputándose la representación de los trabajadores estatales: la Asociación de Trabajadores del Estado (ATE) y la Unión del Personal Civil de la Nación (UPCN). Ambos sindicatos poseen enormes diferencias en términos de posiciones ideológicas, alianzas políticas 
$y$ formas de representación y de acción ${ }^{18}$. Así, hasta la vigencia de la normativa recién mencionada que habilita formalmente la representación gremial de los trabajadores contratados, los dos sindicatos llevaron adelante posiciones disimiles. ATE luego de algunas dudas iniciales respecto a la composición del plantel de contratados, incorporó las demandas de estos trabajadores a sus reivindicaciones e incluso les permitió su afiliación y participación como delegados $y$ en las comisiones internas. UPCN por el contrario, negó una y otra cosa hasta que fue legalmente posible.

\section{CONCLUSIÓN}

El proceso de vulnerabilidad general del trabajo que han vivenciado las sociedades occidentales en las últimas décadas, conforma una realidad muy profunda que se presenta difícil de revertir. El abordaje de la precarización laboral a partir de las múltiples dimensiones en que puede presentarse permite tomar nota de lo extendido y complejo de su desarrollo, sin limitar el análisis a aquellos trabajadores que poseen contratos temporarios o trabajos no registrados; análisis que arroja luz sobre la situación general de la relación entre capital y trabajo.

En este artículo, nos hemos remitido a un caso particular, que posee elementos comunes con el resto de los universos laborales así como algunas singularidades que hemos registrado. En ese sentido, la realidad actual e histórica de la Administración Pública Argentina considerada como espacio de trabajo, aparece como sumamente compleja. El conjunto de reformas del Estado llevadas adelante fundamentalmente durante la década del noventa, constituyen un punto ( $y$ un proceso) de inflexión en las condiciones laborales del conjunto de los trabajadores del Estado. Despidos, retiros voluntarios, congelamiento de la planta permanente, incorporación y promoción masiva de contratos de variadas características, descuentos salariales, etc. conformaron el paquete de medidas que en mayor o menor cuantía alteraron el funcionamiento de la APN, durante los años noventa.

A lo largo de este trabajo se intentó articular dos lógicas que signaron este proceso, una de carácter empírico/conceptual y otra de carácter histórico. Con referencia a la primera pretendimos mostrar los límites de una concepción rígida de la precariedad laboral que distingue compartimentos estancos en los cuales se ubican las diferentes categorías de trabajadores según sus tipos de empleo. La posibilidad de asumir la precariedad a partir de las ocho dimensiones de precariedad aquí relevadas, permite establecer diferencias entre aquellos que cuentan con contratos de tiempo limitado. Asimismo, hay que mencionar que la productividad política del concepto de precariedad laboral se potencia frente a la posibilidad de vislumbrar niveles de vulnerabilidad a lo largo de todo el universo de trabajadores.

Desde el punto de vista histórico, se intentó dar cuenta también de la fragmentación de los colectivos de trabajo que el proceso de heterogeneización de los vínculos de empleo trajo aparejada, fundamentalmente (aunque no solo) entre los trabajadores de la planta permanente $y$ los contratados. El inicial proceso creciente de diferenciación de grupos laboral se ha ingresado en los últimos años (fundamentalmente desde 2005 a la fecha) en un recorrido inverso, en el cual se vienen achicando las diferencias $y$ acercando las visiones $y$ disposiciones de los distintos trabajadores (respecto de los "otros" y de sí mismos) reduciendo considerablemente el conflicto (explícito o latente) de nivel horizontal.

En resumen, al interior de un espacio de trabajo pueden encontrarse conviviendo distintos niveles de precarización y/o vulnerabilidad laboral, variación que puede también desarrollarse a lo largo del plano temporal como se observó en el caso analizado aquí. En consecuencia, se vuelve ostensible la complejidad de esta problemática para definirla de un modo fijo y estanco, o para pensarla simplemente en términos de presencia o ausencia de precariedad, ante la existencia de una realidad mucho más compleja y dinámica. 


\section{BIBLIOGRAFÍA CITADA}

Battistini, Osvaldo. "Flexibilización laboral en Argentina. Un camino hacia la precarización y la desocupación". Revista Venezolana de Gerencia. Maracaibo, 2001.

Bonofiglio, Nicolás; Fernández, Ana. "Sí, señor. Precarización y flexibilización laboral en la década del noventa". VI Congreso Nacional de Estudios del Trabajo. Buenos Aires. Argentina, 2003.

Bouffartigue, Paul. "Précarités professionnelles et action collective. La forme syndicale à l'épreuve". Mimeo. LEST, 2007.

Caire, Guy. "Precarisation des emplois et regulation du marche du travail". Sociologie du travail 2. París, 1982.

Cano, Ernest. "La lógica de la precariedad laboral: el caso de la industria valenciana del mueble". Relaciones Laborales 13. 1998: 207-227.

Capón Filas, Rodolfo. Derecho del trabajo. Platense. La Plata, 1994.

CENDA. "El trabajo en Argentina, condiciones y perspectivas". Informe trimestral 11. 2007. En: <www.cenda.org.ar> [consultada diciembre 2009].

Cingolani, Patrik. La precarité. París: PUF, 2005.

Córdova, Efrén. "Relaciones laborales en la función pública de América Latina”. Revista Internacional del Trabajo 99 (3). 1980. pp. 320.

Coriat, Benjamin. Pensar al revés. Trabajo y organización en la empresa japonesa. España, Siglo XXI editores: 1992.

De la Garza Toledo, E. Los estudios laborales en América Latina al inicio del siglo XXI.
2007. En: <http://docencia.izt.uam.mx/ egt/main.htm> [consultada mayo 2009].

Dessors, Dominique y Ghio Bailly, Marie (comp.). Organización del trabajo y salud. Asociación Trabajo y Sociedad. Programa de Investigaciones Económicas sobre Tecnología, Trabajo y Empleo (CONICET). Buenos Aires: Ed. Lumen Humanitas, 1998.

Diana Menéndez, Nicolás. "La trama compleja de la acción sindical: los casos de ATE y UPCN". Revista Trabajo y Sociedad 10. Universidad de Santiago del Estero, 2008.

Diana Menéndez, Nicolás. "Miradas cruzadas: concepciones y creencias de los dirigentes sindicales en torno a la representación en el Estado". Revista Argentina de Sociología (en prensa). Argentina: CPS, 2010.

Feldman, Silvio y Galin, Pedro. "Nota introductoria". Pedro Galin y Marta Novick (eds.). La precarización del empleo en la Argentina. Buenos Aires. CEAL, 1990: 8-16.

Galin, Pedro y Novick, Marta. La precarización del empleo en la Argentina. Buenos Aires: CEAL, 1990.

Lindenboin, Javier. La precariedad en la Argentina al término del siglo $X X$. 2001. En: <www.cdi.mecon.gov.ar/ biblio/docelec/ceded/lindenboim2.pdf> [consultada abril 2009].

Marshall, Adriana. “¿Contrataciones flexibles o trabajo precario? El empleo temporario y a tiempo parcial". Galin y Novick (comps.). La precarización del empleo en la Argentina. Buenos Aires: Trabajo y Sociedad, 1990.

Meik, Moisés y Zas, Oscar. "Desregulación y flexibilidad normativa de la protección en el ordenamiento laboral argentino". 
Pedro Galin y Marta Novick (eds.). La precarización del empleo en la Argentina. Buenos Aires: CEAL, 1990.

Novick, Marta. "La organización del trabajo en América Latina”. E. de la Garza (comp.). Tratado Latinoamericano de Sociología del Trabajo. México: FCE, 2000.

Offe, C. y Wiesenthal, H. "Dos lógicas de la acción colectiva". La gestión política. España: MTyss, 1992.

Orlansky, Dora; Makon, Ana y Ortiz, Victoria. "Tendencias y cambios posdevaluación en el empleo público". Ponencia presentada en el VII Congreso ASET. Buenos Aires, 2007.

Orsatti, Álvaro. Trabajo precario: antecedentes para su definición desde la OIT. Buenos Aires: MTyss, 1989.

Paugam, Serge. Le salarié de la précarité. París: Ed. PUF, 2000.

Pok, Cintia. "Precariedad laboral: personificaciones sociales en la frontera de la estructura de empleo". Documento de trabajo 29. Buenos Aires: CEIL-PIETTE, 1992.
Poulantzas, Nikos. Estado, poder y socialismo. Siglo XXI, 1980.

Rodgers, G. "El debate sobre el trabajo precario en Europa Occidental". Rodgers, G. y Rodgers, P. (comps.). El trabajo precario en la regulación del mercado laboral: crecimiento del empleo atípico en Europa Occidental. Madrid: MTyss, 1992.

Salvia, Agustín. "Mercados duales y subdesarrollo en la Argentina: fragmentación y precarización de la estructura social del trabajo". VI Congreso Nacional de Estudios del Trabajo. Buenos Aires. Argentina, 2003.

OTROS

Asociación de Trabajadores del Estado: Estatuto.

Subsecretaría de la Gestión Pública. En: <www. sgp.gov.ar> [consultada junio 2010].

Ministerio de Economía. En: <http://www2. mecon.gov.ar/hacienda/ocupacion/ principal.htm $>$ [consultada julio 2010]. 